\title{
Clinical, immunological and bacteriological characteristics of H7N9 patients nosocomially co-infected by Acinetobacter Baumannii: a case control study
}

William J. Liu ${ }^{1,2+}$, Rongrong Zou ${ }^{1 \dagger}$, Yongfei Hu ${ }^{3+}$, Min Zhao ${ }^{3}$, Chuansong Quan², Shuguang Tan ${ }^{3}$, Kai Luo ,

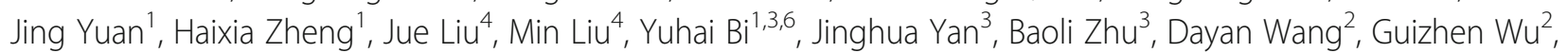
Lei Liu' ${ }^{1}$ Kwok-Yung Yuen ${ }^{5}$, George F. Gao ${ }^{1,2,3,6^{*}}$ and Yingxia Liu ${ }^{1^{*}}$

\begin{abstract}
Background: Bacterial co-infection of patients suffering from influenza pneumonia is a key element that increases morbidity and mortality. The occurrence of Acinetobacter baumannii co-infection in patients with avian influenza A (H7N9) virus infection has been described as one of the most prevalent bacterial co-infections. However, the clinical and laboratory features of this entity of H7N9 and A. baumannii co-infection have not been systematically investigated.

Methods: We collected clinical and laboratory data from laboratory-confirmed H7N9 cases co-infected by A. baumannii. H7N9 patients without bacterial co-infection and patients with A. baumannii-related pneumonia in the same hospital during the same period were recruited as controls. The antibiotic resistance features and the corresponding genome determinants of $A$. baumannii and the immune responses of the patients were tested through the respiratory and peripheral blood specimens.

Results: Invasive mechanical ventilation was the most significant risk factor for the nosocomial A. baumannii co-infection in H7N9 patients. The co-infection resulted in severe clinical manifestation which was associated with the dysregulation of immune responses including deranged T-cell counts, antigen-specific T-cell responses and plasma cytokines. The emergence of genome variations of extensively drug-resistant A. baumannii associated with acquired polymyxin resistance contributed to the fatal outcome of a co-infected patient.
\end{abstract}

Conclusions: The co-infection of H7N9 patients by extensively drug-resistant A. baumannii with H7N9 infection is an important issue which deserves attention. The dysfunctions of immune responses were associated with the co-infection and were correlated with the disease severity. These data provide useful reference for the diagnosis and treatment of H7N9 infection.

Keywords: Avian influenza A(H7N9) virus, Acinetobacter baumannii, Extensively drug-resistant bacteria, Nosocomial infection, Pneumonia, Immune responses

\footnotetext{
* Correspondence: gaofu@chinacdc.cn; yingxialiu@hotmail.com

${ }^{\dagger}$ William J. Liu, Rongrong Zou and Yongfei Hu contributed equally to this work.

Kwok-Yung Yuen, George F. Gao and Yingxia Liu jointly supervised this work.

'Shenzhen Key Laboratory of Pathogen and Immunity, State Key Discipline of Infectious Disease, Shenzhen Third People's Hospital, Shenzhen 518112,

China

Full list of author information is available at the end of the article
}

(c) The Author(s). 2018 Open Access This article is distributed under the terms of the Creative Commons Attribution 4.0 International License (http://creativecommons.org/licenses/by/4.0/), which permits unrestricted use, distribution, and reproduction in any medium, provided you give appropriate credit to the original author(s) and the source, provide a link to the Creative Commons license, and indicate if changes were made. The Creative Commons Public Domain Dedication waiver (http://creativecommons.org/publicdomain/zero/1.0/) applies to the data made available in this article, unless otherwise stated. 


\section{Background}

Although influenza viruses by itself can cause highly fatal primary influenza pneumonia, the excess mortality rates during influenza pandemics is mainly caused by secondary bacterial pneumonia [1]. Epidemiologic evidence suggests that $95 \%$ and $70 \%$ of deaths during the influenza pandemic of 1918-19 and 1957-58, respectively, were due to bacterial pneumonia [2]. During the first season (between May 2009 and August 2009) of 2009 pandemic H1N1 influenza, 29\% of fatal cases in the United States were associated with a secondary bacterial infection, which was also correlated with the severity of the pneumonia [3]. In hospitalized patients with seasonal influenza, sepsis with or without bacteremia were one of the severe complications, and associated with increased duration of hospitalization days and the requirement of intensive care [4]. The secondary bacterial pneumonia was also observed in patients infected by avian influenza viruses including H5N1 and H7N9 [5]. However, the frequency of secondary bacterial respiratory infection and its effect on the disease severity of avian influenza virus-infected human cases were still lacking.

Acinetobacter baumannii is one of the major opportunistic pathogens that have been implicated in various nosocomial infections [6]. The most frequent clinical manifestation of nosocomial $A$. baumannii infection is ventilator-associated pneumonia [7]. Although the clinical impact of nosocomial A. baumannii infection has been a matter of continuing debate as many patients with severe influenza had major underlying diseases [8], it has been concluded that $A$. baumannii infection was associated with an increase in attributable mortality, ranging from 7.8 to $23 \%$ [9]. A. baumannii is attracting much attention owing to the increase in antimicrobial resistance and occurrence of strains that are resistant to virtually all available drugs [10]. The rapid global emergence of multidrug-resistant A. baumannii (MDR-AB) strains resistant to all $\beta$-lactams, including carbapenems, illustrated the potential of this organism to respond swiftly to changes in selective environmental pressure [11]. Though it is still very rare, recently, resistance to polymyxins has also been described [12], which leads to a pandrug-resistant $A$. baumannii (PDR-AB) that can be fully refractory to the currently available antimicrobial armamentarium.

From March 2013, hundreds of human cases infected by avian influenza A (H7N9) virus with a mortality of $40 \%$ were reported and the epidemic situation seems more severe currently with over 100 new cases within December 2016 (http://www.who.int/influenza). Among the H7N9 patients complicated by bacterial infections, MDR-AB was the most common etiology for secondary bacterial pneumonia revealed by independent descriptive studies. Herein, we studied the occurrence of secondary infection by $A$. Baumannii in these severe H7N9 patients. We sought to investigate the role of dysfunctional immunity in the pathogenesis of severe pneumonia in H7N9 patients nosocomially co-infected with $A$. baumannii. Our study may improve the understanding of the high pathogenicity of H7N9 in humans and provide useful recommendations for the clinical diagnosis and treatment.

\section{Methods}

\section{The patients}

From December 2013 to April 2014, 24 patients hospitalized for H7N9 influenza in the Shenzhen Third People's Hospital, Guangdong, China, which is the designated for H7N9 patients in Shenzhen. The laboratory confirmation of H7N9 virus infection were performed using the protocols as described previously $[13,14]$. Briefly, the H7N9 infections of patients were confirmed by real-time RT-PCR assay using influenza subtype-specific primers. Real-time RT-PCR experiments were performed using the RNA which was extracted from the samples using the RNeasy Mini Kit (QIAGEN, Germany). The patients who were diagnosed in other hospitals in Shenzhen were also transferred to our hospital. Meanwhile, we made a surveillance of $A$. baumannii in the hospital. The diagnosis of nosocomial pneumonia by $A$. baumannii was based on the clinical signs of the patients and confirmed by sputum culture and (or) blood culture according to standard microbiological criteria [15]. The patients without typical clinical manifestations were excluded as bacterial colonization of A. baumannii. Among the 24 H7N9 patients, 9 patients were diagnosed as secondary infection by $A$. baumannii (H7N9-A. baumannii or H7N9-AB case group). Thirteen H7N9 patients without secondary bacterial infection with complete clinical data were termed as H7N9 controls. Fifteen patients with hospital acquired pneumonia due to $A$. baumannii, but H7N9 negative in the same period of our hospital, were also recruited as A. baumannii controls. The Declaration of Helsinki was strictly followed. The methods were carried out in accordance with the approved guidelines. We obtained written informed consent from all of the participants or their guardians.

\section{Clinical data investigation}

Data collection (including information prior to and during hospitalization until discharge or death) started immediately after admission and continued on a daily basis. The clinical data were integrated by two independent inputs and checked and verified by the third party. Antimicrobial resistance was determined according to National Committee for Clinical Laboratory Standards guidelines [16]. 


\section{The meta-analyses}

We screened the previous studies on the clinical descriptions of H7N9 patients published from April 2013 to December 2016, through the US National Library of Medicine National Institutes of Health (www.ncbi.nlm. nih.gov). Eleven publications which described the secondary bacterial infections of H7N9 patients were summarized (Table 1).

\section{Inflammatory mediator tests}

We collected the plasma of H7N9-A. baumannii co-infected cases and H7N9 controls on the first day of hospitalization and every seven days afterwards. The levels of different cytokines and chemokines were measured with the Bio-Plex ProTM Human Cytokine Array Kits on a Luminex200TM (Luminex Multiplexing Instrument, Merck Millipore) following the manufacturers' instructions. The raw data were analyzed using xPONENT 3.1 software (Merck Millipore). Plasmas from seven healthy individuals were used as controls.

\section{H7N9-specific T cell response tests}

The CTL epitope-specific response was measured by performing IFN- $\gamma$ ELISPOT assays as described previously [17]. Previously identified HLA class I-restricted epitopes (8-11 residues) of influenza virus were retrieved from published data $[17,18]$. Conserved peptides between H7N9 and 2009pH1N1 were mixed into a conserved peptide pool as described in reference (JID reference) [19]. Mutated HLA class I-restricted peptides between H7N9 and 2009pH1N1 were considered as H7N9-specific peptide pools (JID reference). The number of spots was determined using an automatic ELISPOT reader and image analysis software (Cellular Technology Limited).

\section{A. baumannii genome sequencing and analyzing}

The genomes of the A. baumannii isolates SMGC-AB1 and SMGC-AB2 were sequenced by Illumina HiSeq sequencing platform according to manufacturer's instructions. The 150-bp length pair-end raw reads were first filtered to remove low-quality reads using the DynamicTrim and LengthSort Perl scripts within SolexaQA [20] and then assembled and gap closed using SOAPdenovo2 program (http://soap.genomics.org.cn) [21]. The comparative genome analysis and SNP calling were performed using Mauve software [22]. The draft genomes were annotated using the RAST program (rapid annotation using subsystem technology) [23]. For SNP-based phylogenetic analysis, the whole-genome alignment and SNP calling were performed using Mugsy [24]. The antibiotic resistance gene information was first summarized based on the Subsystem subcategory "Resistance to antibiotics and toxic compounds" according to RAST annotation. Then, the Comprehensive Antibiotic Resistance Database (CARD) [25] was used for the further searching of resistance genes in the sequenced $A$. baumannii genomes.

\section{Accession codes}

The Whole Genome Shotgun projects of patient B4-derived original extensively drug-resistant (XDR) $A$. baumannii SMGC-AB1 and subsequently polymyxin resistant, i.e. a pandrug-resistant isolate (PDR) named $A$. baumannii SMGC-AB2 have been deposited at DDBJ/ ENA/GenBank under the accessions NIBI00000000 and NIBH00000000, respectively.

\section{Statistics}

The Student's $t$-test or $\chi^{2}$-test was used to determine differences between two groups. The Pearson correlation coefficient and Spearman rank correlation coefficient were used for linear correlation analysis. We calculated

Table 1 Mutation information in A. baumannii SMGC-AB2 isolated from H7N9 patient B4 ${ }^{a}$

\begin{tabular}{|c|c|c|c|c|c|c|c|}
\hline No. & Gene Annotation & ORF length & position (NT) & mutation (NT) & AA length & position (AA) & $\overline{\text { substitute }(\mathrm{AA})}$ \\
\hline 1 & putative permease, YjgP/YjgQ family protein & $1101 \mathrm{bp}$ & 164 & $G \rightarrow A$ & 366 & 55 & $\mathrm{Arg} \rightarrow \mathrm{His}$ \\
\hline 2 & histidine kinase; PmrB & 1335 & 704 & $C \rightarrow T$ & 444 & 235 & Thr $\rightarrow \| l e$ \\
\hline 3 & $\begin{array}{l}\text { Lipid A phosphoethanolamine transferase, associated with } \\
\text { polymyxin resistance, PmrC }\end{array}$ & 1647 & 1598 & $A \rightarrow C$ & 548 & 533 & Lys $\rightarrow$ Thr \\
\hline 4 & NAD-dependent aldehyde dehydrogenase & 1575 & 421 & $C \rightarrow T$ & 524 & 141 & Leu $\rightarrow$ Leu \\
\hline 5 & $\begin{array}{l}\text { Large repetitive protein, type I secretion C-terminal target } \\
\text { domain protein }\end{array}$ & 6657 & 547 & $A \rightarrow G$ & 2218 & 183 & $\mathrm{Val} \rightarrow \| \mathrm{e}$ \\
\hline 6 & $\begin{array}{l}\text { Large repetitive protein, type I secretion C-terminal target } \\
\text { domain protein }\end{array}$ & 6657 & 874 & $\mathrm{G} \rightarrow \mathrm{A}$ & 2218 & 292 & $\mathrm{Val} \rightarrow \mathrm{lle}$ \\
\hline 7 & $\begin{array}{l}\text { Large repetitive protein, type I secretion C-terminal target } \\
\text { domain protein }\end{array}$ & 6657 & 876 & $\mathrm{~T} \rightarrow \mathrm{C}$ & 2218 & 292 & $\mathrm{Val} \rightarrow$ lle \\
\hline 8 & Contig C497 & 8383 (positi & & $\mathrm{C} \rightarrow \mathrm{T}$ & Non-codin & g region & \\
\hline
\end{tabular}

${ }^{\mathrm{a}}$ The reference sequence is $A$. baumannii SMGC-AB1 isolated from H7N9 patient B4 isolated on Day 21 after disease onset. $A$. baumannii SMGC-AB2 was isolated from this patient B4 on Day 26 after disease onset 
receiver operating characteristic curves for predictive analysis. Logistic regression was used for multivariate analysis of the risk factors. These statistical analyses were performed with SPSS 16.0 for Windows (SPSS, Inc.). A $P$-value $<0.05$ was considered statistically significant.

\section{Results}

The incidence of $A$. baumannii in H7N9 patients

We have done the meta analyses of the previous studies on the clinical descriptions of H7N9 patients (Additional file 1: Table S1). We found the overall incidence of $A$. baumannii among the reported H7N9 patients was $19.0 \%(37 / 195)$. For the 11 A. baumannii cases whose outcome were available, 10 patients $(90.9 \%)$ was reported fatal, much higher than the crude mortality rate of $32.9 \%(56 / 170)$ amongst H7N9 patients. Most of the identified A. baumannii were MDR-AB, and with one case of PDR-AB.
From December 2013 to April 2014, 24 patients infected by H7N9 virus were hospitalized in the Shenzhen Third People's Hospital, Guangdong, China. We found that there were two peaks of hospital admission for H7N9 patients (Fig. 1a, Additional file 2: Table S2 and Additional file 3: Table S3). One is from the late December 2013 to early February 2014 and the second peak is from the early March to early April 2014. Meanwhile, we made a surveillance of positive $A$. baumannii isolation in our hospital and found that the weekly incidence of $A$. baumannii was correlated with the hospitalization of H7N9 patients (Fig. 1b). Among the 24 H7N9 patients, 9 patients $(37.5 \%)$ were diagnosed as secondary bacterial pneumonia caused by A. baumannii (H7N9-A. baumannii group) during the hospitalization or on retrospective testing (Fig. 1c). Meanwhile, in the same Infectious Disease Unit which was specially designated for H7N9 admission, none of the other 353 patients

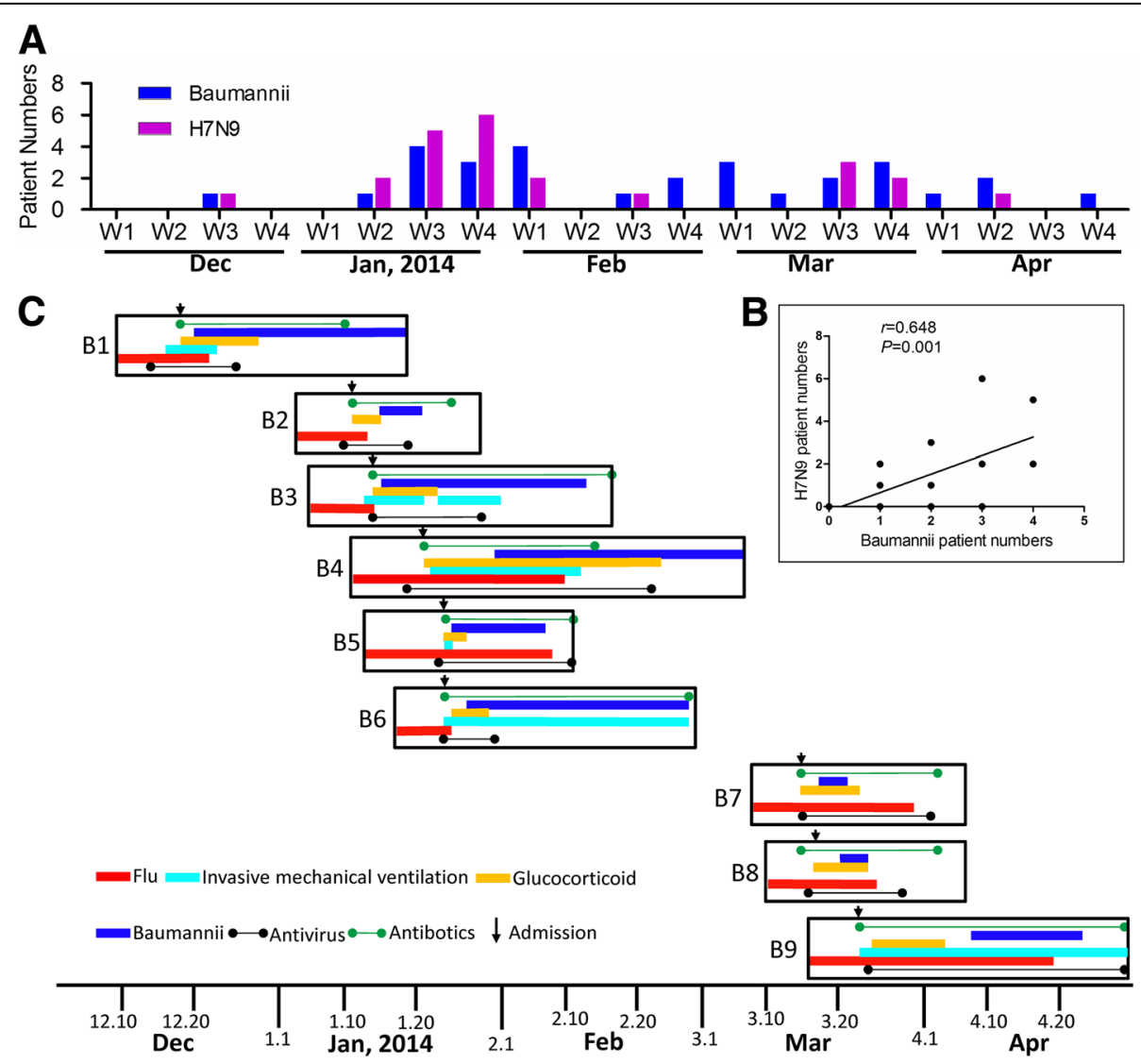

Fig. 1 The occurrence of nosocomial A. baumannii infection in H7N9 patients during the timeline of the 2013-2014 H7N9 epidemic wave in the hospital. a. The weekly reported cases infected by H7N9 and A. baumannii, respectively, from December 2013 to April 2014 in Shenzhen Third People's Hospital, Guangdong China. The first week to the fourth week of each month were denoted as W1 to W4. $\mathbf{b}$. The linear correlation of weekly reported numbers of H7N9 patients and A. baumannii patients. $\mathbf{c}$. The timeline of the laboratory test and clinical treatment of the H7N9 and A. baumannii co-infected patients. The corresponding dates for timeline were shown below as the horizontal axis. Flu: The persistent period for positive result of H7N9 RNA detected by RT-PCR; Baumannii: The period for A. baumannii positive; Admission: the date for the admission of the patient; Antivirus: the period for the anti-influenza drug therapy; Antibiotics: the period for the antibiotic use; Glucocorticoid: the period for glucocorticiod pulse therapy; Invasive mechanical ventilation: the period for the manipulation of invasive mechanical ventilation 
without H7N9 were diagnosed as A. baumannii infection. This may exclude the possibility that any poor infection control led to a high frequency of this $A$. baumannii infection in H7N9 patients.

\section{The risk factors for $A$. baumannii co-infection in H7N9 patients}

The median duration from admission to the detection of A. baumannii was three days. Based on the time line of the clinical interventions, we found that most of the $A$. baumannii co-infection with H7N9 occurred after the application of invasive mechanical ventilation, antibiotics and broad-spectrum immune intervention drugs such as glucocorticoid (methylprednisolone). Furthermore, the risk factors for the secondary infection of $A$. baumannii in the H7N9 patients were analyzed. We found that the H7N9-A. baumannii co-infected patients have a significantly longer duration of invasive mechanical ventilation (Fig. 2a), antibiotics use (Fig. 2b) and glucocorticoid use (Fig. 2c) compared to the 13 H7N9 patients without secondary bacterial infection (H7N9 group, the clinical data for the left two patients are not completed). The total dosages of glucocorticoid and globulin were also higher among H7N9 patients co-infected by $A$. baumannii compared to other H7N9 patients without secondary bacterial infection (Fig. 2d). The multivariate analysis considering the mutual effects of different risk factors showed that invasive mechanical ventilation was still the risk factor for the A. baumannii coinfection in H7N9 patients (Additional file 4: Table S4 and Additional file 5: Table S5).

\section{The clinical impact of $A$. baumannii on H7N9 infection}

The basic demographic characteristics of H7N9 patients co-infected with $A$. baumannii are similar to H7N9 control patients without secondary infection (Additional file 2: Table S2). The average age of H7N9-A. baumannii co-infection group was higher than the H7N9 controls but without significance $(61.8 \pm 18.3$ vs $48.2 \pm 14.9, P>$ $0.05)$. No significant differences were found for the clinical manifestations at the time of admission of H7N9-A.baumannii cases compared to the H7N9 controls, including the oxygenation index $\left(\mathrm{PaO}_{2} / \mathrm{FiO}_{2}\right)(136.3 \pm 60.0$ vs 217.3 $\pm 145.0, \mathrm{P}>0.05$ ) (Additional file 3: Table S3 and Fig. 3a). However, the lowest oxygenation index was lower and the time for the restoring of oxygenation index was longer during the hospitalization process of the H7N9-A. baumannii cases than the H7N9 controls $(66.8 \pm 31.5$ vs $163.9 \pm 122.1$ ) (Fig. 3b). Furthermore, the recovered times for the abnormal oxygenation indexes in H7N9-A. baumannii cases were longer than the H7N9 controls (Fig. $3 c$ ), indicating a more severe pulmonary ventilation
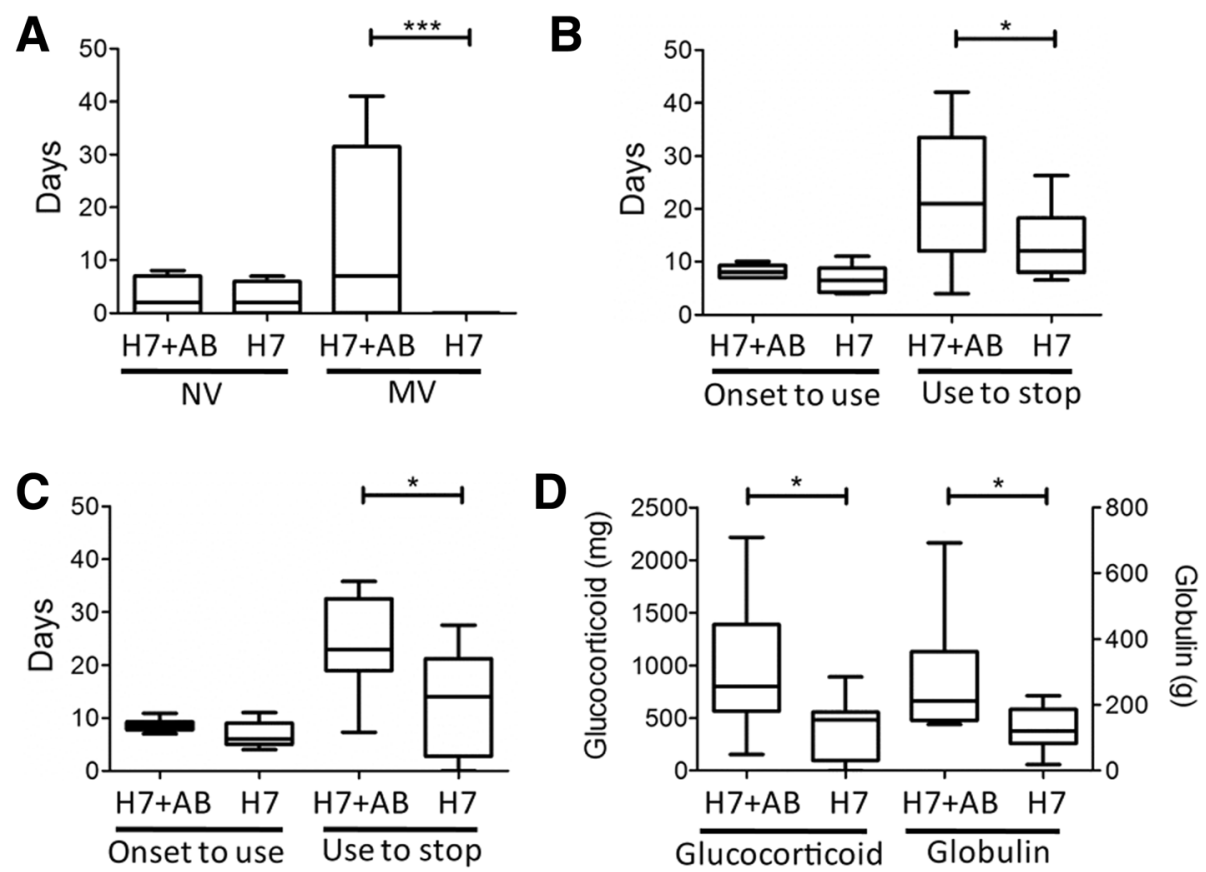

Fig. 2 The risk factors for H7N9-A. baumannii co-infection. a. The comparison of the manipulation of non-invasive ventilation (NV) and invasive mechanical ventilation (MV) between H7N9-A. baumannii co-infected cases (H7N9 + AB) and H7N9 control patients without bacterial co-infection (H7N9). b. The time from the disease onset to the use of antibiotics (Onset to use) and the total time for the antibiotic use (Use to stop) is compared between $\mathrm{H7N} 9+\mathrm{AB}$ cases and H7N9 controls. c. The time from the disease onset to the use of glucocorticoid (Onset to use) and the total time for the glucocorticoid use (Use to stop) is compared between H7N9+ AB cases and H7N9 controls. $\mathbf{d}$. The total dosages for glucocorticoid and gamma globulin compared between between H7N9 + AB cases and H7N9 controls 


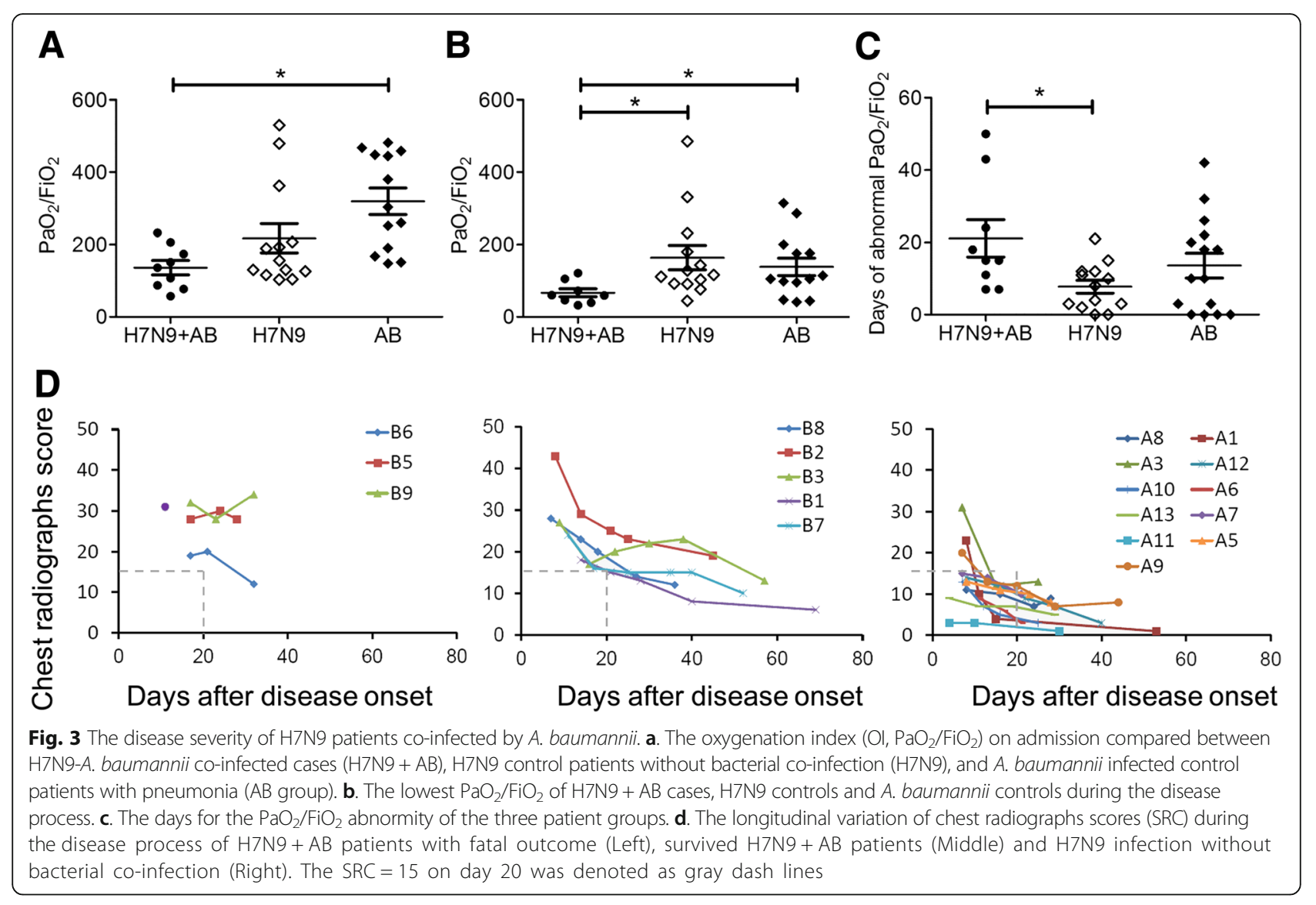

disorder of the H7N9-A. baumannii cases after onset of co-infection by $A$. baumannii. The evaluation of chest radiograph score (SRC) which reflects the lung injury during the infection indicates a prolonged high SRC in the H7N9-A. baumannii cases (Fig. 3d). All the SRC of the H7N9-A. baumannii cases are still $>15$ on day 20 after disease onset, while the SRC of most H7N9 controls without co-infection decreased to $<15$ on day 20 after disease onset. The H7N9-A. baumannii cases had longer time for fever duration $(14.3 \pm 9.2$ vs $8.1 \pm 2.5)$ and the length of hospital are also longer than the H7N9 controls without co-infection $(30.0 \pm 11.2$ vs $15.5 \pm 7.1)$. All H7N9 controls without co-infection survived, but four of the nine (44.4\%) H7N9-A. baumannii patients died. In the H7N9-A. baumannii cases, the patients who died had persistent abnormal oxygenation index compared to that of a gradual improvement in the survivors (Additional file 6: Figure S1).

\section{Dysfunctional immunity in co-infected patients}

IL-6 and IL-8 are two major representative cytokines which can reflect the disease severity during the acute phase of H7N9 as reported previously [26]. Furthermore, the cytokines IL-6 and IL-8 among H7N9 patients without $A$. baumannii presented a decreasing trend during the disease course (Fig. 4a and b). In contrast, both the IL-6 and IL-8 among the plasma of the H7N9-A. baumannii patients increased in the third week after disease onset and remained higher than the H7N9 patients until the fifth week after disease onset or death.

The T-cell counts correlated with the lung function of the H7N9 patients (Additional file 7: Figure S2). We next investigated the T-cell subgroups of the H7N9-A. baumannii cases. We found that the patients who had co-infection by $A$. baumannii have fewer $\mathrm{CD} 4^{+}$and $\mathrm{CD} 8^{+}$ T-cells, compared to H7N9 controls without co-infection, and also lower than the A. baumannii controls without H7N9 infection (Fig. 4c-f). Generally, the normalization time for the $\mathrm{CD} 4^{+}$T-cell count among the H7N9-A. baumannii cases are also longer than those of the H7N9 controls and A. baumannii controls (Fig. 4e). For the longitudinal profile of the lymphocyte count, fatal patients with H7N9-A. baumannii co-infection had a consistently low level of both $\mathrm{CD} 4^{+}$and $\mathrm{CD}^{+} \mathrm{T}$-cell counts, while the survived H7N9-A. baumannii patients have gradual normalization of T-cell counts but still much slower than the H7N9 controls without co-infection (Additional file 8: Figure S3).

We performed biostatistical analyses to further identify correlations between immune factors as of H7N9-A. 


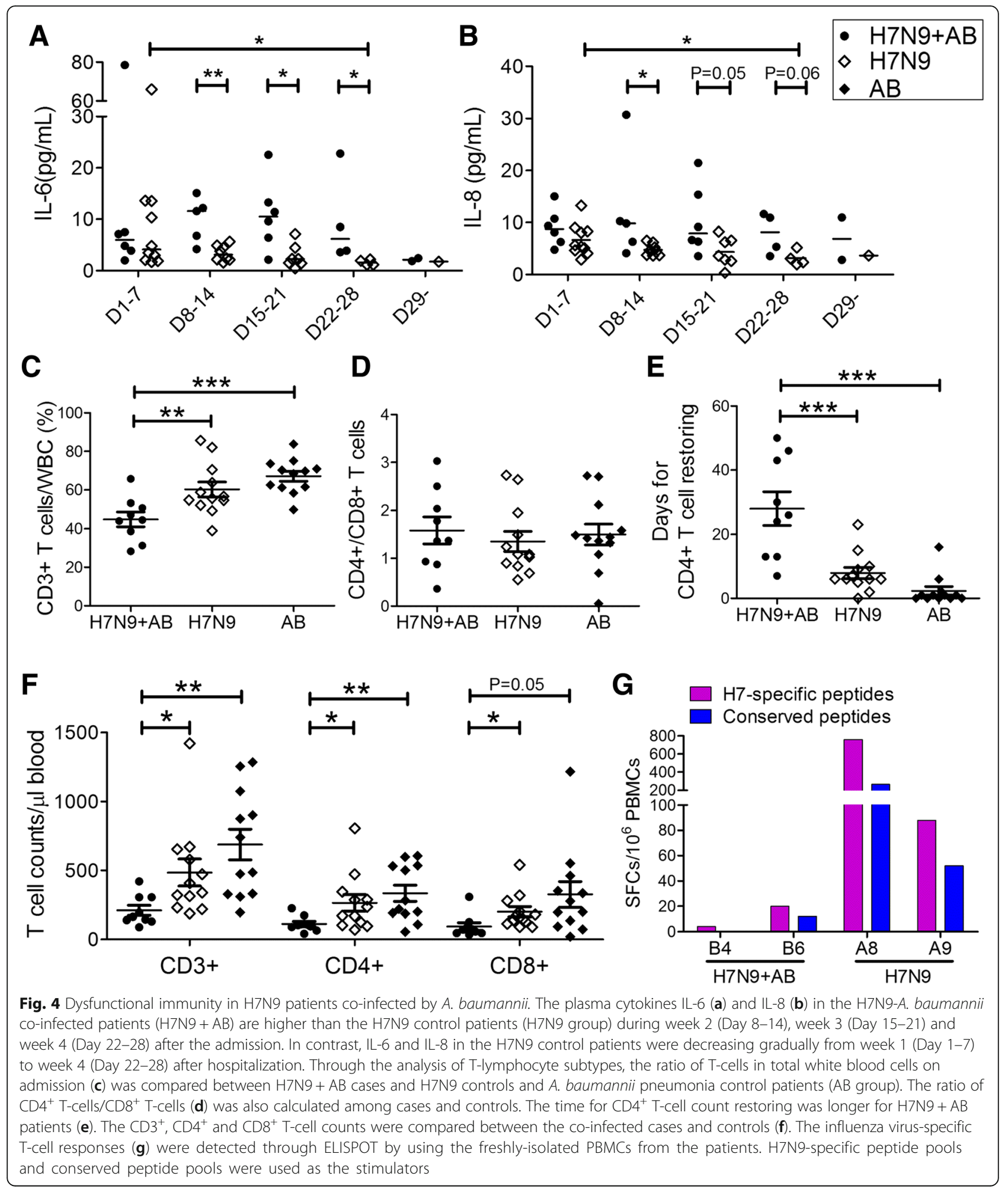

baumannii co-infection. We first used SPSS software to calculate its predictive value for co-infection. The area under the curve (AUC) of plasma IL-6 and IL- 8 levels were 0.927 and 0.850 respectively during the second week of hospitalization among H7N9-infected patients, and were 0.881 and 0.833 during the third week (Additional file 9: Figure S4). In addition, the $\mathrm{CD}^{+} \mathrm{T}$-cell counts (AUC: 0.846), CD8 ${ }^{+}$T-cell counts (AUC: 0.812 ) on admission and the restoring time for the abnormal $\mathrm{CD} 4^{+} \mathrm{T}$-cell counts (AUC: 0.876 ) were all predictive for the H7N9-A. 
baumannii co-infection. Additionally, the traditional biomarkers of bacterial infection, procalcitonin and C-reactive protein levels in blood fluctuated during the disease process, but the two indicators of fatal cases with H7N9-A. baumannii co-infection remained at a higher level during a longer period compared to survived cases (Additional file 10: Figure S5 and Additional file 11: Figure S6). We also used multivariate analysis in logistic regression to further analyze the independent predictor for $A$. baumannii co-infection among patients with H7N9 infections (Additional file 4: Table S4 and Additional file 5: Table S5). CD4 ${ }^{+}$T-cell counts in blood harvested on the day of admission were found to be an independent indicator among the clinical parameters of H7N9-infected patients including procalcitonin and $\mathrm{C}$-reactive protein.

\section{Lower virus-specific T-cell responses amongst H7N9-A. baumannii co-infected patients}

The influenza virus-specific T-cell tests through the ELIPOST assays by the stimulation of H7N9-derived peptides or conserved peptides, indicated that H7N9-A. baumannii patients had a weak $\mathrm{CD}^{+}$T-cell responses to H7N9 virus compared to H7N9 controls without co-infection (Fig. 4g).

\section{The antibiotic resistance features of the $A$. baumannii noscomially acquired by H7N9 patients}

After an average time of 6 days of invasive ventilation, the H7N9 patients were detected to be $A$. baumannii positive. According to standard drug resistance definition [27], the original isolates from six patients were identified as extensively drug-resistant (XDR). As the A. baumannii strains were susceptible to polymyxin, the patients were then treated with polymyxin B with an average total dosage of 14.7 million units for an average 10.5 days. Notably, for patient B4, after polymyxin treatment for five days, the original XDR isolate SMGC-AB1 became polymyxin resistant, i.e. a pandrug-resistant isolate (PDR) named SMGC-AB2 (Table 1, Additional file 12: Table S6).

\section{Rapid emergence of a pandrug-resistant isolate featured by candidate polymyxin resistance-associated mutations} To gain insights into the genetic features of the H7N9 patient infected with $A$. baumannii, the whole genomes of both SMGC-AB1 and SMGC-AB2 were sequenced. SNP-based phylogenic analysis showed that SMGC-AB1 was clustered into the European clone (EC) II group, including MDR-ZJ06, MDR-TJ, BJAB0868 and BJAB07104 from Mainland China, TYTH-1 and TCDC-AB0715 from China Taiwan and AB1656-2 from Korean (Additional file 13: Figure S7). The SMGC-AB1 genome harbored a total of 57 genes belonging to Subsystem subcategory "Resistance to antibiotics and toxic compounds" according to RAST annotation (Additional file
14: Figure S8). Notably, SMGC-AB1 encodes 6 $\beta$-lactamases and 2 aminoglycoside adenylyl-transferases.

When comparing the genome of SMGC-AB1 with its polymyxin resistance descendant, SMGC-AB2, only $8 \mathrm{nu}$ cleotide point mutations were identified in SMGC-AB2, 7 of which were located in coding regions and 6 lead to nonsynonymous mutation (Table 1). The 1st mutation was located in a putative permease protein encoding gene belonging to YjgP/YjgQ family (now LptF/LptG), which has been identified to be a component of $\mathrm{ABC}$ transporter for lipopolysaccharide transport to the cell surface [28]. Notably, the 2nd and 3rd mutations were located in the pmrCAB operon, a gene cluster encoding the PmrC phosphoethanolamine phosphotransferase involved in polymyxin resistance [29]; mutations in genes of $p m r B$ and pmrC leading to $\mathrm{Thr} \rightarrow \mathrm{Ile}$ (T235I) and Lys $\rightarrow$ Thr (L533 T) substitution, respectively. The 5th to 7th mutations causing Val $\rightarrow$ Ile (V183I and V292I) are all located in a large repetitive protein harboring type I secretion C-terminal target domain. The 4th mutation resulting in no amino acid substitution appeared in a gene encoding NAD-dependent aldehyde dehydrogenase, and the 8th lies in the non-coding region.

\section{Discussion}

It is known that secondary bacterial pneumonia during influenza virus infection is a major cause for high mortality of severe or fatal cases. Compared with the dominant bacterial infection of Streptococcus pneumoniae and Staphylococcus aureus in other influenza virus pandemics [30], A. baumannii is the most commonly encountered pathogen in sputum or endotracheal samples in the H7N9 virus infected clinical cases [31]. Here, we presented a typical cohort of the H7N9 patients confected by $A$. baumannii and elucidated their dysfunctional immune responses. Furthermore, the genome features of the A. baumannii and particularly the genome variations contributed to the acquired polymyxin resistance which might have led to the fatal outcome of this patient.

The role of the hospital environment as a reservoir for A. baumannii has been well defined [32]. Hospital equipment, especially the medical equipment for invasive mechanical ventilation, is one of the important risk factors that predispose individuals to the acquisition of, and infection with, A. baumannii [6]. Other risk factors include prior antibiotic use and prior use of broad-spectrum drugs [33]. In our study, H7N9 patients co-infected by A. baumannii have a longer use of antibiotics, glucocorticoid, and the invasive mechanical ventilation, all of which may predispose to the occurrence of the $A$. baumannii co-infection in these patients.

In fact, influenza virus infection itself also enhances the susceptibility of the patients to secondary bacterial 
infection by its impacts on the inflammatory signals and function of early innate immune defense [34]. It is known that levels of inflammatory mediators such as IL-6 and IL-18 were higher in the patients with co-infection of influenza virus and bacteria than in patients with bacterial pneumonia or influenza virus infection alone [35]. Concordantly, in our study, the IL-6 and IL-8 increased to a higher level in the H7N9-A. baumannii co-infected patients compared to H7N9 patients without bacterial infection.

Influenza A infection could also increase susceptibility to secondary bacterial pneumonia by dysregulation of different innate immune cells. The influenza virus can inhibit Th17 immunity by the induction of type I IFNs [36] and IL-27 [37], and also can inhibit neutrophil attraction through the mediation of protein Setdb2 [38] and the production of IL-10 [39]. In our study, we found that the H7N9 patients with a persistently low level of $\mathrm{CD}^{+}$and $\mathrm{CD}^{+}{ }^{+}$-cell population, especially hyporesponsive antigen-specific T-cell responses, are susceptible to the A. baumannii infection and the subsequent fatal outcome. This may indicate a T-cell anergy after the avian H7N9 influenza virus infection as previously defined in severe $\mathrm{H} 1 \mathrm{~N} 1$ infected patients [40].

It is surprising that SMGC-AB1 quickly acquired resistance to polymyxin, an antibiotic that is considered to be the last hope to cope with MDR and or XDR gram-negative bacteria. Polymyxins are cyclic cationic peptides with a long hydrophobic tail that interact with the lipid A moiety of lipopolysaccharide (LPS) to disrupt the integrity of outer membranes of gram-negative bacteria. Three major resistance mechanisms to polymyxin in bacteria have been identified: modification of the bacterial outer membrane lipopolysaccharide, proteolytic cleavage of the drug and activation of broad-spectrum efflux pumps, among which modification of lipid A of LPS regulated by the two-component regulatory system PmrAB has been frequently reported in A. baumannii [41]. It is reported that a single mutation in $\operatorname{pmr} B$ gene causing T235I amino acid substitution can lead to 16-fold increases in polymyxin B MIC in A. baumannii [29]. In this study, we found that the SMGC-AB2 harbors a mutation in $p m r B$ gene exactly resulting in T235I amino acid substitution; we believe that this mutation plays a decisive role for this isolate to resist polymyxin. As demonstrated, mutations in the pmrA or $p m r B$ genes usually result in the constitutive expression of $p m r C$, thus leading to LPS modification and reduction of the affinity of polymyxins [42]. In clinical resistant isolates, similar mutations have also been found in $p m r C$ gene. However, in SMGC-AB2, the mutation located at the end of the $\mathrm{C}$-terminus of pmrC protein (L533 T), beyond the functional sulfatase domain (aa 237-532), may not contribute to the resistance. It is reported that A. baumannii can also develop resistance to polymyxin by mutating genes responsible for LPS production [43]. Though no mutations were found in the biosynthesis pathway of LPS in SMGC-AB2, a mutation appeared in a pupative permease (Table 1, mutation No. 1) that probably functions in LPS transport. We speculate that this mutation may cause the decrease of LPS production, thus leading to the decrease of the drug target, which may also play a secondary role in the resistance phenotype of SMGC-AB2. Finally, we also found three mutations in a large repetitive protein harboring type I secretion C-terminal target domain leading to two amino acid substitutions ( Val $\rightarrow$ Ile); whether and how this protein and the mutations are involved in the polymyxin resistance in SMGC-AB2 needs further investigations.

In conclusion, we described the occurrence of secondary infection of $A$. baumannii and its impacts on the disease severity in H7N9 patients. The dysfunctional immunity in the H7N9 patients correlated to the A. baumannii co-infection. Furthermore, the genome variations of $A$. baumannii contributed to the acquired polymyxin resistance in the patient with fatal outcome. We suggest that the enhancement of the anti-nosocomial infection measures for the prevention of $A$. baumannii in the H7N9 patients with risk factors for secondary infections, and the early administration of appropriate antibiotic regimen when such co-infection is detected by frequent microbiological testing.

\section{Conclusions}

Invasive mechanical ventilation is the most significant risk factor for the nosocomial A. baumannii co-infection in in patients with avian influenza A (H7N9) virus infection. The occurrence of H7N9 co-infection with A. baumannii is a key factor for the severity of the patients, with a manifestation of lower oxygenation indexes and chest radiograph scores. Dysregulation of immune responses in the H7N9 patients correlates to the susceptibility of $A$. baumannii co-infection and severe clinical manifestation. Novel polymyxin resistance-associated genome variations of $A$. baumannii could quickly emerge during the disease process and may contribute to fatal outcome of the H7N9-A. baumannii co-infected patients. Both the immune and bacterial features in the patients may contribute to and act as biomarkers for the severe pneumonia and fatal outcome of the co-infection. These conclusions shed light on the pathomechanism, diagnosis and treatment of $\mathrm{H7N} 9$ and bacterial co-infections. 


\section{Additional files}

Additional file 1: Table $\mathbf{S} 1$. The meta analyses of $A$. baumannii infection in H7N9 patients. (DOCX 59 kb)

Additional file 2: Table S2. Basic characters of the patients. (DOCX $34 \mathrm{~kb}$ ) Additional file 3: Table S3. Clinical presentation and main lab-findings on admission. (DOCX $34 \mathrm{~kb}$ )

Additional file 4: Table S4. The multivariate analysis of the risk factors of H7N9 patients co-infected by A. baumannii $(n=13)$ compared to H7N9 control patients $(n=9)$. (DOCX $32 \mathrm{~kb}$ )

Additional file 5: Table S5. The multivariate analysis of the clinical risk factors and indicators of H7N9 patients co-infected by A. baumannii ( $n=13)$ compared to the group $(n=24)$ combined of H7N9 control and $A$. baumannii control patients. (DOCX $32 \mathrm{~kb}$ )

Additional file 6: Figure S1. The longitudinal variation of oxygenation index $\left(\mathrm{PaO}_{2} / \mathrm{FiO}_{2}\right)$ in the $\mathrm{H} 7 \mathrm{~N} 9$ patients co-infected by $A$. baumannii. (PDF $190 \mathrm{~kb}$ )

Additional file 7: Figure S2. The association of T-cell counts with oxygenation index $\left(\mathrm{PaO}_{2} / \mathrm{FiO}_{2}\right)$ in the $\mathrm{H} 7 \mathrm{~N} 9$ patients. (PDF $237 \mathrm{~kb}$ )

Additional file 8: Figure S3. The longitudinal trends of $\mathrm{CD}^{+}{ }^{+} \mathrm{T}$-cell counts, CD4 ${ }^{+} \mathrm{T}$-cell counts and $\mathrm{CD} 8^{+} \mathrm{T}$-cell counts among the H7N9 patients. (PDF $210 \mathrm{~kb}$ )

Additional file 9: Figure S4. ROC curve of the plasma levels of cytokines and T-cell characteristics in H7N9 patients. (PDF $431 \mathrm{~kb}$ )

Additional file 10: Figure S5. The longitudinal variation of $P C T$ in the H7N9 patients co-infected by A. baumannii. (PDF 185 kb)

Additional file 11: Figure S6. The longitudinal variation of CRP in the H7N9 patients co-infected by A. baumannii. (PDF 188 kb)

Additional file 12: Table S6. Antibiotic susceptibility profiles for $A$. baumannii SMGC-AB1 and SMGC-AB2. (DOCX $32 \mathrm{~kb}$ )

Additional file 13: Figure S7. Phylogenetic position of $A$. baumannii SMGC-AB1 and its polysaccharide antigen gene clusters. (PDF $624 \mathrm{~kb}$ )

Additional file 14: Figure S8. Comparison of gene distribution in Subsystem subcategory "Resistance to antibiotics and toxic compounds". (PDF 337 kb)

\section{Acknowledgements}

We thank Dr. Jianfang Zhou and Dr. Hui Li for their helpful suggestions.

\section{Ethics approval and consent to participates}

The study was approved by the Ethics Review Committee of Shenzhen Third People's Hospital, Shenzhen China. The study was conducted in accordance with the principles of the Declaration of Helsinki and the standards of good clinical practice (as defined by the International Conference on Harmonization). The written informed consent from all of the participants or their guardians were obtained.

\section{Funding}

This study was supported by grants from National Natural Science Foundation of China (81373141, 81330082 and 81401312), the Intramural Special Grant for Influenza Virus Research from the Chinese Academy of Science (KJZD-EW-L15) the Shenzhen Science and Technology Research and Development Project (JCYJ20160427151920801) and the National Key Research and Development Program of China (2016YFC1200800). GFG is a leading principal investigator of the National Natural Science Foundation of China Innovative Research Group (81621091). YH is a member of the Youth Innovation Promotion Association of Chinese Academy of Sciences (2015069). The funding sources had no role in the study design, data collection and analysis, decision to publish, or preparation of the manuscript.

\section{Availability of data and materials}

The datasets generated and/or analysed during the current study are available at DDBJ/ENA/GenBank under the accessions NIBI00000000 and NIBH00000000, respectively.

\section{Authors' contributions}

WJL, GFG, YL designed research studies; WL, YH, MZ, CQ, ST conducted the experiments; $R Z, K L, J Y, H Z J L, M L$ collected and analyzed the clinical data; YB, JY, BZ, DW, GW, LL supported the materials; WJL, YH, K-YY, GFG, YL wrote the paper. All authors read and approved the final manuscript.

\section{Consent for publication}

Not applicable.

\section{Competing interests}

The authors declare that they have no competing interests.

\section{Publisher's Note}

Springer Nature remains neutral with regard to jurisdictional claims in published maps and institutional affiliations.

\section{Author details}

'Shenzhen Key Laboratory of Pathogen and Immunity, State Key Discipline of Infectious Disease, Shenzhen Third People's Hospital, Shenzhen 518112 , China. ${ }^{2} \mathrm{NHC}$ Key Laboratory of Medical Virology and Viral Diseases, National Institute for Viral Disease Control and Prevention, Chinese Center for Disease Control and Prevention, Beijing, China. ${ }^{3}$ CAS Key Laboratory of Pathogenic Microbiology and Immunology, Institute of Microbiology, Chinese Academy of Sciences (CAS), Beijing, China. ${ }^{4}$ Department of Epidemiology and Biostatistics, School of Public Health, Peking University, Beijing, China. ${ }^{5}$ State Key Laboratory for Emerging Infectious Diseases, The University of Hong Kong, Special Administration Region, Hong Kong, China. ${ }^{6}$ Center for Influenza Research and Early-Warning (CASCIRE), Chinese Academy of Sciences, Beijing, China.

Received: 23 November 2017 Accepted: 16 October 2018

Published online: 14 December 2018

\section{References}

1. Kash JC, Taubenberger JK. The role of viral, host, and secondary bacterial factors in influenza pathogenesis. Am J Pathol. 2015;185(6):1528-36.

2. Morens DM, Taubenberger JK, Fauci AS. Predominant role of bacterial pneumonia as a cause of death in pandemic influenza: implications for pandemic influenza preparedness. J Infect Dis. 2008;198(7):962-70.

3. Weinberger DM, Simonsen L, Jordan R, Steiner C, Miller M, Viboud C. Impact of the 2009 influenza pandemic on pneumococcal pneumonia hospitalizations in the United States. J Infect Dis. 2012;205(3):458-65.

4. Dawood FS, Chaves SS, Perez A, Reingold A, Meek J, Farley MM, Ryan P, Lynfield R, Morin C, Baumbach J, et al. Complications and associated bacterial coinfections among children hospitalized with seasonal or pandemic influenza, United States, 2003-2010. J Infect Dis. 2014;209(5): 686-94

5. Yuen KY, Chan PK, Peiris M, Tsang DN, Que TL, Shortridge KF, Cheung PT, To WK, Ho ET, Sung R, et al. Clinical features and rapid viral diagnosis of human disease associated with avian influenza a H5N1 virus. Lancet. 1998; 351(9101):467-71

6. Garnacho-Montero J, Ortiz-Leyba C, Fernandez-Hinojosa E, Aldabo-Pallas T, Cayuela A, Marquez-Vacaro JA, Garcia-Curiel A, Jimenez-Jimenez FJ. Acinetobacter baumannii ventilator-associated pneumonia: epidemiological and clinical findings. Intens Care Med. 2005;31(5):649-55.

7. Trouillet JL, Chastre J, Vuagnat A, Joly-Guillou ML, Combaux D, Dombret MC, Gibert C. Ventilator-associated pneumonia caused by potentially drugresistant bacteria. Am J Respir Crit Care Med. 1998;157(2):531-9.

8. Garnacho J, Sole-Violan J, Sa-Borges M, Diaz E, Rello J. Clinical impact of pneumonia caused by Acinetobacter baumannii in intubated patients: a matched cohort study. Crit Care Med. 2003;31(10):2478-82.

9. Falagas ME, Rafailidis PI. Attributable mortality of Acinetobacter baumannii: no longer a controversial issue. Crit Care. 2007;11(3):134.

10. Perez F, Hujer AM, Hujer KM, Decker BK, Rather PN, Bonomo RA. Global challenge of multidrug-resistant Acinetobacter baumannii. Antimicrob Agent Chemother. 2007:51(10):3471-84

11. Wu W, He Y, Lu J, Lu Y, Wu J, Liu Y. Transition of blaOXA-58-like to blaOXA23-like in Acinetobacter baumannii clinical isolates in southern China: an 8year study. PLoS One. 2015;10(9):e0137174. 
12. Li J, Rayner CR, Nation RL, Owen RJ, Spelman D, Tan KE, Liolios L. Heteroresistance to colistin in multidrug-resistant Acinetobacter baumannii. Antimicrob Agent Chemother. 2006;50(9):2946-50.

13. Gao R, Cao B, Hu Y, Feng Z, Wang D, Hu W, Chen J, Jie Z, Qiu H, Xu K, et al. Human infection with a novel avian-origin influenza a (H7N9) virus. New Engl J Med. 2013;368(20):1888-97.

14. Li J, Yu X, Pu X, Xie L, Sun Y, Xiao H, Wang F, Din H, Wu Y, Liu D, et al. Environmental connections of novel avian-origin H7N9 influenza virus infection and virus adaptation to the human. Sci China Life Sci. 2013;56(6):485-92.

15. Calandra T, Cohen J. International Sepsis forum definition of infection in the ICUCC: the international sepsis forum consensus conference on definitions of infection in the intensive care unit. Crit Care Med. 2005;33(7):1538-48.

16. Patel JB, Cockerill FR, Bradford PA, Eliopoulos GM, Hindler JA, Jenkins SG, Lewis JS, Limbago B, Miller LA, Nicolau DP et al: Performance standards for antimicrobial susceptibility testing; twenty-fourth informational supplement. CLSI Standard Antimicrob Suscep Test 2015, 35(3):231.

17. Liu J, Wu B, Zhang S, Tan S, Sun Y, Chen Z, Qin Y, Sun M, Shi G, Wu Y, et al. Conserved epitopes dominate cross-CD8+ T-cell responses against influenza a H1N1 virus among Asian populations. Eur J Immunol. 2013;43(8):2055-69.

18. Vita R, Zarebski L, Greenbaum JA, Emami H, Hoof I, Salimi N, Damle R, Sette A, Peters B. The immune epitope. database 2.0. Nucleic Acid Res. 2010; 38(Database issue):D854-62.

19. Liu WJ, Tan S, Zhao M, Quan C, Bi Y, Wu Y, Zhang S, Zhang H, Xiao H, Qi J, et al. Cross-Immunities against Avian Influenza H7N9 Virus in the Healthy Population Affected by Antigenicity-Dependent Substitutions. J Infect Dis. 2016;214:1937-46

20. Cox MP, Peterson DA, Biggs PJ. SolexaQA: at-a-glance quality assessment of Illumina second-generation sequencing data. BMC Bioinformatics. 2010;11:485.

21. Luo R, Liu B, Xie Y, Li Z, Huang W, Yuan J, He G, Chen Y, Pan Q, Liu Y, et al. SOAPdenovo2: an empirically improved memory-efficient short-read de novo assembler. Gigascience. 1(1):18.

22. Darling AC, Mau B, Blattner FR, Perna NT. Mauve: multiple alignment of conserved genomic sequence with rearrangements. Genome Res. 2004; 14(7):1394-403.

23. Aziz RK, Bartels D, Best AA, DeJongh M, Disz T, Edwards RA, Formsma K, Gerdes S, Glass EM, Kubal M, et al. The RAST server: rapid annotations using subsystems technology. BMC Genomics. 2008;9:75.

24. Angiuoli SV, Salzberg SL. Mugsy: fast multiple alignment of closely related whole genomes. Bioinformatics. 2011;27(3):334-42.

25. McArthur AG, Waglechner N, Nizam F, Yan A, Azad MA, Baylay AJ, Bhullar K, Canova MJ, De Pascale G, Ejim L, et al. The comprehensive antibiotic resistance database. Antimicrob Agent Chemother. 2013;57(7):3348-57.

26. Guo J, Huang F, Liu J, Chen Y, Wang W, Cao B, Zou Z, Liu S, Pan J, Bao C, et al. The serum profile of Hypercytokinemia factors identified in H7N9infected patients can predict fatal outcomes. Sci Rep. 2015;5:10942.

27. Magiorakos AP, Srinivasan A, Carey RB, Carmeli Y, Falagas ME, Giske CG, Harbarth S, Hindler JF, Kahlmeter G, Olsson-Liljequist B, et al. Multidrugresistant, extensively drug-resistant and pandrug-resistant bacteria: an international expert proposal for interim standard definitions for acquired resistance. Clin Microbiol Infect. 2012;18(3):268-81.

28. Ruiz N, Gronenberg LS, Kahne D, Silhavy TJ. Identification of two innermembrane proteins required for the transport of lipopolysaccharide to the outer membrane of Escherichia coli. Proc Natl Acad Sci U S A. 2008;105(14):5537-42.

29. Arroyo LA, Herrera CM, Fernandez L, Hankins JV, Trent MS, Hancock RE. The pmrCAB operon mediates polymyxin resistance in Acinetobacter baumannii ATCC 17978 and clinical isolates through phosphoethanolamine modification of lipid a. Antimicrob Agent Chemother. 2011;55(8):3743-51.

30. McCullers JA. Insights into the interaction between influenza virus and pneumococcus. Clin Microbiol Rev. 2006;19(3):571-82.

31. Gao HN, Lu HZ, Cao B, Du B, Shang H, Gan JH, Lu SH, Yang YD, Fang Q Shen $Y Z$, et al. Clinical findings in 111 cases of influenza a (H7N9) virus infection. N Engl J Med. 2013;368(24):2277-85.

32. Peleg $A Y$, Seifert $H$, Paterson DL. Acinetobacter baumannii: emergence of a successful pathogen. Clin Microbiol Rev. 2008;21(3):538-82.

33. Dijkshoorn L, Nemec A, Seifert H. An increasing threat in hospitals: multidrugresistant Acinetobacter baumannii. Nat Rev Microbiol. 2007;5(12):939-51.

34. McCullers JA. The co-pathogenesis of influenza viruses with bacteria in the lung. Nat Rev Microbiol. 2014;12(4):252-62.

35. Rosseau S, Hocke A, Mollenkopf H, Schmeck B, Suttorp N, Kaufmann SH, Zerrahn J. Comparative transcriptional profiling of the lung reveals shared and distinct features of Streptococcus pneumoniae and influenza a virus infection. Immunology. 2007;120(3):380-91.

36. Li W, Moltedo B, Moran TM. Type I interferon induction during influenza virus infection increases susceptibility to secondary Streptococcus pneumoniae infection by negative regulation of gammadelta T cells. J Virol. 2012;86(22):12304-12

37. Robinson KM, Lee B, Scheller EV, Mandalapu S, Enelow RI, Kolls JK, Alcorn JF. The role of IL-27 in susceptibility to post-influenza Staphylococcus aureus pneumonia. Respir Res. 2015;16:10.

38. Schliehe C, Flynn EK, Vilagos B, Richson U, Swaminathan S, Bosnjak B, Bauer L, Kandasamy RK, Griesshammer IM, Kosack L, et al. The methyltransferase Setdb2 mediates virus-induced susceptibility to bacterial superinfection. Nat Immunol. 2015;16(1):67-74.

39. van der Sluijs KF, van Elden $L$, Nijhuis $M$, Schuurman R, Pater JM, Florquin $S$, Goldman M, Jansen HM, Lutter R, van der Poll T. IL-10 is an important mediator of the enhanced susceptibility to pneumococcal pneumonia after influenza infection. J Immunol. 2004;172(12):7603-9.

40. Agrati C, Gioia C, Lalle E, Cimini E, Castilletti C, Armignacco O, Lauria FN, Ferraro F, Antonini M, Ippolito G, et al. Association of profoundly impaired immune competence in $\mathrm{H} 1 \mathrm{~N} 1 \mathrm{~V}$-infected patients with a severe or fatal clinical course. J Infect Dis. 2010;202(5):681-9.

41. Adams MD, Nickel GC, Bajaksouzian S, Lavender H, Murthy AR, Jacobs MR, Bonomo RA. Resistance to colistin in Acinetobacter baumannii associated with mutations in the PmrAB two-component system. Antimicrob Agent Chemother. 2009;53(9):3628-34

42. Beceiro A, Llobet E, Aranda J, Bengoechea JA, Doumith M, Hornsey M, Dhanji H, Chart H, Bou G, Livermore DM, et al. Phosphoethanolamine modification of lipid a in colistin-resistant variants of Acinetobacter baumannii mediated by the pmrAB two-component regulatory system. Antimicrob Agent Chemother. 2011;55(7):3370-9.

43. Moffatt JH, Harper M, Harrison P, Hale JD, Vinogradov E, Seemann T, Henry R, Crane B, St Michael F, Cox AD, et al. Colistin resistance in Acinetobacter baumannii is mediated by complete loss of lipopolysaccharide production. Antimicrob Agent Chemother. 2010;54(12):4971-7.

\section{Ready to submit your research? Choose BMC and benefit from:}

- fast, convenient online submission

- thorough peer review by experienced researchers in your field

- rapid publication on acceptance

- support for research data, including large and complex data types

- gold Open Access which fosters wider collaboration and increased citations

- maximum visibility for your research: over $100 \mathrm{M}$ website views per year

At $\mathrm{BMC}$, research is always in progress.

Learn more biomedcentral.com/submissions 